Andrea Padoan, Chiara Cosma, Ada Aita, Filippo Navaglia, Daniela Basso, Gianfranco Giannella and Mario Plebani*

\title{
Hyris bCUBE SARS-CoV-2 rapid molecular saliva testing: a POCT innovation on its way
}

https://doi.org/10.1515/cclm-2022-0008

Received January 4, 2022; accepted January 11, 2022; published online January 18, 2022

\section{Abstract}

Objectives: The reliable identification of individuals with SARS-CoV-2 infection is the cornerstone for containing viral spread. Rapid molecular point-of-care testing (POCT) of saliva might reduce analysis time, thus increasing the efficacy of contact tracing. In this study, a new POCT RT-PCR assay for the detection of SARS-CoV-2 RNA in saliva was evaluated and compared with an already validated CE-IVD method.

Methods: An evaluation was made of 160 left-over salivary samples (27 frozen, kept at $-80{ }^{\circ} \mathrm{C}$ and 133 fresh), collected using Salivette (Sarstedt, Germany). Samples were analyzed by TaqPath COVID-19 CE-IVD RT-PCR kit, QuantStudio5 Real-Time (Applied Biosystems, USA) (TaqPath) and bKIT Virus Finder COVID-19 Saliva (Hyris Global Diagnostics, Italy). Performances of three- and fivefold pooling strategies were also evaluated. Blood assay interference in saliva was also tested with Hyris.

Results: On using TaqPath, SARS-CoV-2 positivity was detected in 35 samples. Another 10 positive samples were artificially-generated by blind mixing of positive with negative samples. Hyris positive and negative percentages of agreement were 97.6 (95\% CI: 87.2-99.9\%) and 100 (95\% CI: $97.0-100 \%$ ), respectively. Seventeen positive pools, evaluated for threefold strategy, were all correctly determined by both systems. For the 5-pool strategy, 94.7\% (18/ 19) of samples resulted positive with the Hyris system, and

\footnotetext{
*Corresponding author: Prof. Mario Plebani, MD, Department of Medicine-DIMED, University of Padova, Padova, Italy; and Department of Laboratory Medicine, University-Hospital of Padova (Italy), Via Giustiniani 2, 35128, Padova, Italy, E-mail: mario.plebani@unipd.it. https://orcid.org/0000-0002-0270-1711

Andrea Padoan and Daniela Basso, Department of Medicine-DIMED, University of Padova, Padova, Italy; and Department of Laboratory Medicine, University-Hospital of Padova, Padova, Italy. https://orcid.org/0000-0003-1284-7885 (A. Padoan) Chiara Cosma, Ada Aita and Filippo Navaglia, Department of Laboratory Medicine, University-Hospital of Padova, Padova, Italy Gianfranco Giannella, Hyris Ltd, London, UK
}

$100 \%$ with TaqPath. The presence of $1 \%$ of blood $(v / v)$ in saliva did not interfere with the accuracy of Hyris assay. Conclusions: The sensitivity and specificity of the bKIT Virus Finder COVID-19 Saliva were optimal with respect to TaqPath. In view of the safe and straightforward preanalytical procedure involved, and the small size of the Hyris bCube, the Hyris system can be used for POCT.

Keywords: assay comparison; COVID-19; pooling strategies; rapid molecular testing; saliva; SARS-CoV-2.

\section{Introduction}

The fourth wave of Coronavirus Disease 2019 (COVID-19) has afflicted countries worldwide since late summer 2021. The recent wide spread of SARS-CoV-2 in Autumn 2021 has alerted European countries and, currently, several national governments are reimposing lockdowns, whilst massive testing policies are undergoing in order to limit viral spread and allow appropriate contact-tracing programs [1]. In turn, while the number of test requests has increased, pressure on clinical laboratories has become critical, and alternative testing strategies are urgently needed in order to ensure the timely detection of positive individuals. However, to assure accuracy of results, analytical systems able to detect SARS-CoV-2 with high clinical sensitivity and specificity are mandatory, especially for the screening of asymptomatic subjects [2]. Since rapid antigenic tests do not reliably identify positive individuals, they are unsuitable for use in screening contexts [3]. Differently, the real-time reverse transcription polymerase chain reaction (rRT-PCR) test for SARS-CoV-2 diagnosis has a very high analytical sensitivity [3], and has therefore been endorsed not only for confirming a diagnosis of COVID-19, but also in situations calling for reliable testing, such as screening of healthcare workers and following the pre-hospitalization protocol for surgery candidates [4]. However, since rRT-PCR entails multi-step analysis (including RNA extraction, reverse transcription to cDNA and DNA amplification), it has a long analytical turnaround time, multiple instrumentations usually being required to perform all the required tasks. Hence, the rRT-PCR evaluation of large numbers of samples might call for elevated automation, several instrumentations and 
overnight testing. Rapid rRT-PCR testing for SARS-CoV-2, recently developed to obviate the time-limitation issue, is often based on one-step rRT-PCR (direct) or LAMP technology amplification [5]. RT-PCR and LAMP have also been implemented in point of care (POCT) devices [5], which are more convenient for reaching outpatients and testing sites for COVID-19 outside hospitals. Currently, a total of 17 POCT devices have been granted Emergency Use Authorization (EUA) by the FDA for SARS-CoV-2 testing, demonstrating that this new technology is gaining greater attention [6].

Another possible limitation in successfully achieving SARS-CoV-2 testing is the reluctance of some individuals to undergo nasopharyngeal swab (NPS). Especially in pediatric subjects and in screening programs, the use of saliva for SARS-CoV-2 testing appears a valid and successful strategy [7], due to the possibility of well tolerated auto-collection by means of approved devices.

In this study, we evaluated the clinical performances of a new POCT device, the Hyris system, consisting of the bKIT Virus Finder COVID-19, the bCUBE and the bAPP, in detecting SARS-CoV-2 in salivary samples. The performances were evaluated and compared with those of a reference method, the TaqPath COVID-19 CE-IVD RT-PCR kit, already validated for saliva testing.

\section{Materials and methods}

A total of 160 salivary leftover samples, collected by Salivette (Sarstedt, Nümbrecht, Germany, ref 51.5134) at the University Hospital of Padova, were included in the study. The positivity or negativity of all samples to SARS-CoV-2 were first evaluated by a CE-IVD system (reference method) as follows: $200 \mu \mathrm{L}$ saliva was used to extract SARS-CoV-2 RNA by automated nucleic acid extraction using the Roche MagNA Pure 96 Instrument with the MagNA Pure 96 DNA and Viral NA Small Volume Kit (pathogen universal 200 protocol). RNA was subsequently amplified by QuantStudio ${ }^{\text {TM }}$ five Real-Time PCR Systems (Applied Biosystems, USA) using the TaqPath COVID-19 CE-IVD RT-PCR kit (Life Technology, Applied Biosystems, USA). TaqPath COVID-19 RT-PCR kit has the CE-IVD certification for the usage of salivary samples. Of the 160 samples, 27 were collected and stored immediately at $-80{ }^{\circ} \mathrm{C}$ within $24 \mathrm{~h}$ following collection.

Molecular testing of SARS-CoV-2 by Hyris system [bKIT Virus Finder COVID-19 Saliva, the bCUBE 2.0 system and 16-well cartridge and the bAPP v 1.5.12 (https://bapp.hyris.net/)] was performed in both fresh and frozen samples (after thawing at room temperature for $30 \mathrm{~min}$ ). Briefly, the Hyris system uses $50 \mu \mathrm{L}$ saliva, treated with lysis buffer, heated to $95^{\circ} \mathrm{C}$ for $20 \mathrm{~min}$, and then loaded in a 6 or 16 samples cartridge, which is inserted into the bCUBE instrument for analysis launched by bApp, web software on cloud. One-step RT-PCR amplifies two sequences of SARS-CoV-2 $N$ gene ( $N 1$ and $N 2$ ) and $h R R P 3 O$ (RNase $P$ human gene) in 45 cycles on crude samples.

Fresh specimens were from leftover samples obtained daily during the screening program conducted at the University-Hospital of Padova from November 15th to December 3rd 2021, while frozen samples were from patients hospitalized with moderate or severe COVID-19 disease in September 2021 [8]. To increase the number of positive specimens, a series of 10 positive artificially-generated samples were also tested. These samples were made up as follows: $5 \mu \mathrm{L}$ of two positive samples (chosen blind) were mixed with $40 \mu \mathrm{L}$ of one of the samples found to be negative by both systems, to obtain a total volume of $50 \mu \mathrm{L}$. These artificially generated samples were tested blind by both the Hyris system and the reference method. In the presence of discordant results, samples were retested with both systems; if the volume of a sample was not sufficient for re-testing using both systems, the sample was excluded from the evaluation. Considering a confidence level of $95 \%$, the sample size of 160 specimens adopted in this study with a positive rate of $23.0 \%$, allows the sensitivity values below 0.90 to be significantly different in comparison to a sensitivity of 1.0, whilst specificity values below 0.97 are significantly different in comparison to a specificity of 1.0.

Two saliva pooling strategies (pools of three and five samples) were also tested. To prepare pools of three samples, $10 \mu \mathrm{L}$ of a positive sample were pipetted in a vial containing $40 \mu \mathrm{L}$ negative saliva, consisting of two negative samples ( $20 \mu \mathrm{L}$ each); to prepare pools of five samples, $10 \mu \mathrm{L}$ positive sample were pipetted in a vial containing $40 \mu \mathrm{L}$ negative saliva, consisting of four negative samples. A total of 17 pools were generated by for evaluating threefold pooling, while a total of 19 pools were generated for evaluating fivefold pooling.

Blood interferences were evaluated on three SARS-CoV-2 positive saliva samples and three SARS-CoV-2 negative saliva samples. Blood was assessed at a final concentration of $1 \%(\mathrm{v} / \mathrm{v})$ in the clinical sample.

GraphPad Prism v 9.1 for Windows (GraphPad Software, San Diego, California USA) and PASS 2020 Power Analysis and Sample Size Software (2020) (NCSS, LLC. Kaysville, Utah, USA) were used for statistical analyses.

The study protocol (number 23307) was approved by the Ethics Committee of the University-Hospital of Padova.

\section{Results}

Results obtained on comparing the Hyris system with the reference method are reported in Table 1 . Of the 125 samples found negative by the reference method, three were excluded from the specificity calculation, since specimens were interpreted as indeterminate by the Hyris system, but volumes were too small for retesting. On considering the 35 samples found positive by the reference method, 30 were confirmed positive by the Hyris System, two negative, one inconclusive and two indeterminate. However, for one negative and two indeterminate samples, volumes were inadequate for retesting; the inconclusive sample was probably due to the low viral load: with the reference method, the $N$ gene $\mathrm{Ct}$ threshold was 35.6 , which was very close to the $36 \mathrm{Ct}$, the reference method threshold. The 10 artificially generated positive samples were all found to be positive by both the Hyris system and reference method. Figure 1 reports the $\mathrm{Ct}$ values obtained for $N$ gene ( $N 1$ and $N 2$ primers) of the Hyris system and $N$ gene of the reference method. Determination coefficients $\left(\mathrm{R}^{2}\right)$ were 0.7728 for $N 1$ vs. $N$ and 0.7061 for $N 2$ vs. $N$. 
Table 1: Estimation of Hyris bKIT Virus Finder COVID-19 Saliva performances with respect to the reference method.

\begin{tabular}{llllr}
\hline SARS-CoV-2 & \multicolumn{3}{c}{ Reference method } \\
& & \multicolumn{1}{c}{ TaqPath COVID-19 CE-IVD RT-PCR kit } \\
\cline { 3 - 5 } & & Positive & Negative & Total \\
\hline Hyris bKIT & Positive & 40 & 0 & $40^{\mathrm{b}}$ \\
Virus & Indeterminate/ & $3^{\mathrm{a}}$ & $3^{\mathrm{a}}$ & $6^{\mathrm{a}}$ \\
Finder & inconclusive & & & $123(1)^{\mathrm{b}}$ \\
COVID-19 & Negative & $1(1)^{\mathrm{b}}$ & 122 & 168 \\
Saliva & Total & $44^{\mathrm{a}}(1)^{\mathrm{b}}$ & 125 & \\
& Diagnostic & PPA $^{\mathrm{c}}: 97.6 \%$ & NPA & : $100 \%$ \\
& performances & $(95 \% \mathrm{Cl}:$ & $(95 \% \mathrm{Cl}:$ & \\
& & $87.2-99.9 \%)$ & $97.0-100 \%)$ & \\
\hline
\end{tabular}

${ }^{a}$ Not included in the performance estimation, since volumes were insufficient for retesting and sample degradation could not be ruled out; ${ }^{b}$ negative sample not included in the performance estimation, since volumes were insufficient for retesting and sample degradation could not be ruled out; 'PPA: positive agreement percentage, calculated by TP/(TP + FN); ${ }^{d} \mathrm{NPA}$ : negative agreement percentage.

For the evaluation of pooling strategies performances, a total of 17 pools were generated by pooling 51 specimens (pools of three samples, made up of one positive and two negative saliva samples), while 19 pools were generated by pooling 95 specimens (pools of five samples, made up of one positive and four negative saliva samples). For pools of three samples, all samples (17/17) were correctly identified as positive by the Hyris system; for pools of five samples, $18 / 19(94.7 \%)$ of samples were correctly identified as positive.

The assessment of blood interference with analysis showed that all the three positive and three negative saliva specimens were correctly determined by the Hyris system as expected; hence, $1 \%$ of blood in saliva does not interfere with analytical results. Overall, the number (percentage) of undetermined/inconclusive tests obtained on testing samples with the Hyris system was 6/160 (3.75\%).

\section{Discussion}

Two years after the first announced case of SARS-CoV-2 infection, the COVID-19 pandemic continues to plague countries worldwide. Despite the development of safe and effective vaccines against COVID-19, efficient strategies to reduce community spread of the virus continue to be physical barriers (e.g. face masks and distancing), contact tracing, and screening for the identification of asymptomatic individuals [1]. Molecular detection of SARS-CoV-2 RNA in nasopharyngeal swab (NPS) is considered the "gold standard". However, NPS sampling calls for trained personnel and may be difficult to achieve in some patients, children in particular [9]. In addition, rRT-PCR testing requires specialized laboratories, secure sample handling, and results are released several hours after sampling. Altogether, these factors compromise effective screening, and prolong time-to-result thus delaying the isolation of positive individuals and limiting the efficacy of contact tracing [10]. The use of oral saliva, collected by patients themselves, is well tolerated, increases participation in screening and obviates the need for specialized operators for sample collection [7]. Finally, as demonstrated, saliva is stable for 7-10 days for SARS-CoV-2 molecular testing [11], and also offers reliable results for antigen detection [12].

This study evaluated the diagnostic performances of an innovative POCT device for the molecular determination of SARS-CoV-2 in saliva by RT-PCR.

The Hyris bKIT Virus Finder COVID-19 Saliva is powered by such features as its: 1 ) short (around $2 \mathrm{~h}$ ) analytical turnaround time (TAT); 2) straightforward analytical workflow; 3) very small ultra-portable device and 4) provision of remote cloud-based validation of results, also using artificial intelligence. The limitations of this system are its: 1 ) low throughput ( 6 or 12 samples, depending on cartridge type), and 2) limited detection in salivary sample of 9 copies $/ \mu \mathrm{L}$ (possibly higher than two-step RT-PCR). The Hyris bKIT Virus Finder COVID-19 Saliva has been specifically designed to operate in salivary samples, while the performances of the assay for nasopharyngeal swabs (Hyris bKIT Virus Finder COVID-19) have been already evaluated [13, 14].

Results were evaluated and compared with those of a reference method, the TaqPath COVID-19 CE-IVD RT-PCR kit for saliva. Unlike the Hyris system, which amplified two sequences of the SARS-CoV- $2 N$ gene and $h R R P 30$, the reference method amplified one sequence of each of these genes: $N, E$, $S$ and RdRp as housekeeping. Thus, the comparison of results was performed considering only the $N$ gene of the reference system. The TAT of the methods differs, because the Hyris systems allows a "direct" analysis, thus enabling a fast, straightforward and safe pre-analytical workflow: immediately after collection, saliva is centrifugated, heat inactivated, protease treated and directly seeded in a Hyris analysis cartridge. Overall, the effective time-to-results (including sample preparation) requires $2 \mathrm{~h}$ for Hyris system, while the TaqPath RT-PCR assay requires $4 \mathrm{~h}$. The absence of an RNA extraction step in the Hyris system minimizes the analytical TAT, but causes a shift to a higher $\mathrm{Ct}$, as shown in Figure 1. This limitation is mitigated by the increase of amplification cycles by up to 45 . However, the higher $\mathrm{Ct}$ is also required by the system for automatic results interpretation, which is also supported by artificial intelligence cloud-based tools. 


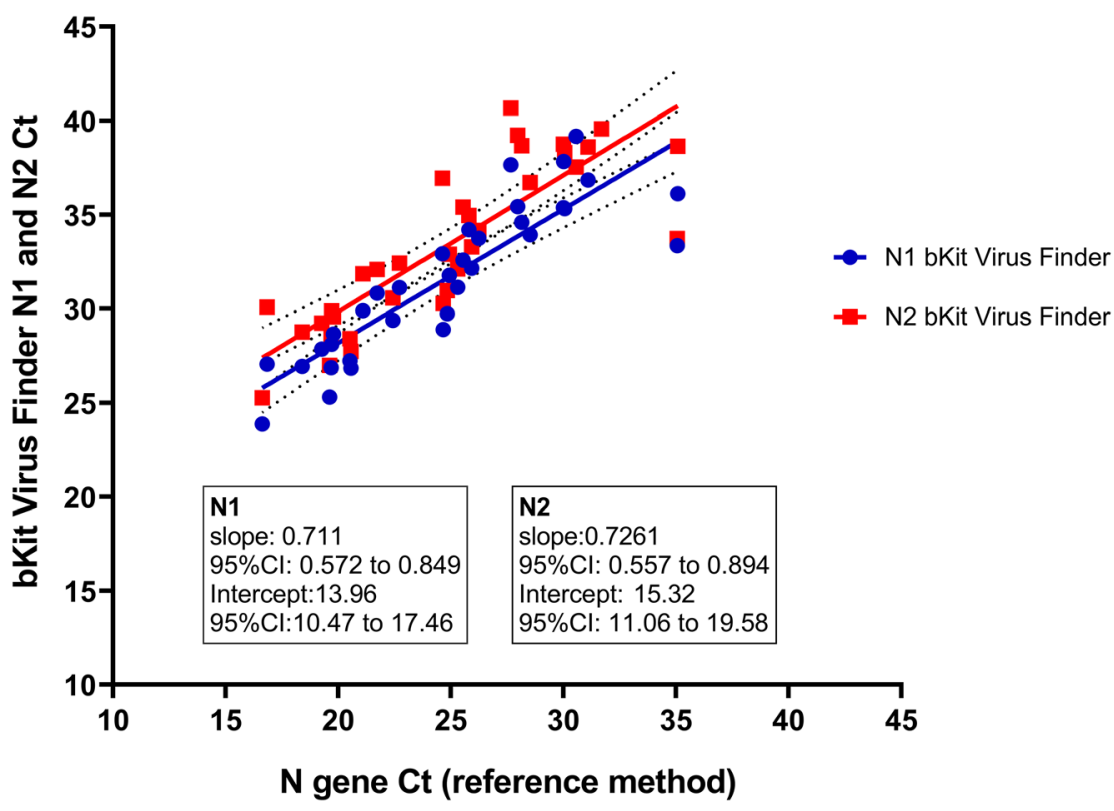

Figure 1: Dotplot reporting the $\mathrm{Ct}$ values obtained for $N$ gene ( $N 1$ and $N 2$ primers) of the Hyris system and $N$ gene of the reference method. Linear regressions and their $95 \% \mathrm{Cl}$ (dotted lines) are also shown.
The results made in the present study (Table 1) demonstrate optimal performances (sensitivity $>95 \%$, and specificity $\cong 100 \%$ ), in accordance with findings made by $\mathrm{Ng}$ et al. [9], who tested a rapid salivary molecular test for SARS-CoV-2 based on LAMP technology. Conversely, according to other Authors, the sensitivity of POCT devices for saliva molecular testing is lower than that of NPS RT-PCR [10]. However, a possible limitation of our study might be that some samples were excluded from the performance estimation, since specimen volumes were small (and retesting with both systems was not possible). Although an uncommon occurrence, nucleic acid degradation in frozen samples could not be ruled out, since SARS-CoV-2 is an RNA virus. In three of the negative samples that were excluded, the lack of housekeeping gene amplification caused indeterminate results.

Further evaluations were made in order to determine the best possible pooling strategy, which was endorsed for facilitating the detection of early community transmission of SARS-CoV-2, and to enable the timely implementation of appropriate infection control measures with a view to containing spread, also with salivary testing $[15,16]$. The findings made in the present study suggest that the best available pooling strategy for the Hyris system is threefold pools, whereas fivefold pools lose sensitivity. The reason for this consideration is that in real-word screening, fivefold pools might be not reliable enough, especially for detecting low viral load cases [17], found in SARS-CoV-2 infected vaccinated individuals [18]. Finally, saliva blood contamination of up to $1 \%$ does not lead to false negative results.
This study presents some limitations, such as the use of three different sample types for improving positive rate and, hence, the study power (saliva fresh, stored and artificially prepared saliva) and the evaluation of interferences that has been tested only with $1 \%$ of blood in saliva.

The results of this study demonstrate that the molecular detection of SARS-CoV-2 in saliva by bKIT Virus Finder COVID-19 Saliva has an optimal performance in terms of sensitivity and specificity with respect to the two-step RT-PCR method TaqPath. The pre-analytical procedure is safe and straightforward, and the Hyris bCube is small, thus supporting the use of this system outside hospitals (e.g. in airports and schools) for screening programs. The artificial intelligence included in this system obviates human error, thus facilitating use, also by personnel other than laboratory technicians.

Acknowledgments: The Authors acknowledge the technical support by Daniela Rinaldi (Laboratory Medicine, University-Hospital of Padova).

Research funding: None declared.

Author contributions: All authors have accepted responsibility for the entire content of this manuscript and approved its submission.

Competing interests: Authors state no conflict of interest. Informed consent: Informed consent was obtained from all individuals included in this study.

Ethical approval: The study protocol (number 23307) was approved by the Ethics Committee of the UniversityHospital of Padova. 


\section{References}

1. Zhou Y, Jiang H, Wang Q, Yang M, Chen Y, Jiang Q. Use of contact tracing, isolation, and mass testing to control transmission of covid-19 in China. BMJ 2021. https://doi.org/10.1136/bmj.n2330 [Epub ahead of print].

2. Gray N, Calleja D, Wimbush A, Miralles-Dolz E, Gray A, De Angelis $M$, et al. Is "no test is better than a bad test"? Impact of diagnostic uncertainty in mass testing on the spread of COVID-19. PLoS One 2020;15:1-18.

3. Dinnes J, Deeks JJ, Berhane S, Taylor M, Adriano A, Davenport C, et al. Rapid, point-of-care antigen and molecular-based tests for diagnosis of SARS-CoV-2 infection. Cochrane Database Syst Rev 2021;3:CD013705.

4. Kahn M, Schuierer L, Bartenschlager C, Zellmer S, Frey R, Freitag M, et al. Performance of antigen testing for diagnosis of COVID-19: a direct comparison of a lateral flow device to nucleic acid amplification based tests. BMC Infect Dis 2021;21:798.

5. Sakthivel D, Delgado-Diaz D, McArthur L, Hopper W, Richards JS, Narh CA. Point-of-Care diagnostic tools for surveillance of SARS-CoV-2 infections. Front Public Health 2021;9:1-9.

6. U.S. Food and Drug Administration. In vitro diagnostics EUA molecular diagnostic tests for SARS-CoV-2; 2021. https://www. fda.gov/medical-devices/coronavirus-disease-2019-covid19-emergency-use-authorizations-medical-devices/in-vitrodiagnostics-euas-molecular-diagnostic-tests-sars-cov-2 [Accessed 29 Dec 2021].

7. Ku CW, Shivani D, Kwan JQT, Loy SL, Erwin C, Ko KKK, et al. Validation of self-collected buccal swab and saliva as a diagnostic tool for COVID-19. Int J Infect Dis 2021;104:255-61.

8. World Health Organization. Clinical management of COVID-19, interim guidance. https://www.who.int/publications/i/item/ clinical-management-of-covid-19 [Accessed 29 Dec 2021].

9. Ng D, Pinharanda A, Vogt MC, Litwin-Kumar A, Stearns K, Thopte U, et al. WHotLAMP: a simple, inexpensive, and sensitive molecular test for the detection of SARS-CoV-2 in saliva. PLoS One 2021;16:1-18.

10. LeGoff J, Kernéis S, Elie C, Mercier-Delarue S, Gastli N, Choupeaux L, et al. Evaluation of a saliva molecular point of care for the detection of SARS-CoV-2 in ambulatory care. Sci Rep 2021;11:1-8.

11. Ott IM, Strine MS, Watkins AE, Boot M, Kalinich CC, Harden CA, et al. Stability of SARS-CoV-2 RNA in nonsupplemented saliva. Emerg Infect Dis 2021;27:1146-50.

12. Basso D, Aita A, Padoan A, Cosma C, Navaglia F, Moz S, et al. Salivary SARS-CoV-2 antigen rapid detection: a prospective cohort study. Clin Chim Acta 2021;517:54-9.

13. Miscio L, Olivieri A, Labonia F, De Feo G, Chiodini P, Portella G, et al. Evaluation of the diagnostic accuracy of a new point-ofcare rapid test for SARS-CoV-2 virus detection. J Transl Med 2020;18:488.

14. Gori Savellini G, Anichini G, Terrosi C, Prathyumnan S, Gandolfo C, Marini S, et al. Comparative performance of a new SARS-CoV-2 rapid detection system. Microbiol Spectr 2021;9:e0020521.

15. Spellberg B, Haddix M, Lee R, Butler-Wu S, Holtom P, Yee H, et al. Community prevalence of SARS-CoV-2 among patients with influenzalike illnesses presenting to a los angeles medical center in march 2020. JAMA 2020;323:1966.

16. Girón-Pérez DA, Ruiz-Manzano RA, Benitez-Trinidad AB, VenturaRamón GH, Covantes-Rosales CE, Ojeda-Durán AJ, et al. Saliva pooling strategy for the large-scale detection of SARS-CoV-2, through working-groups testing of asymptomatic subjects for potential applications in different workplaces. J Occup Environ Med 2021;63:541-7.

17. Mahmoud SA, Ibrahim E, Thakre B, Teddy JG, Raheja P, Ganesan S, et al. Evaluation of pooling of samples for testing SARS-CoV-2 for mass screening of COVID-19. BMC Infect Dis 2021;21:360.

18. Levine-Tiefenbrun M, Yelin I, Katz R, Herzel E, Golan Z, Schreiber L, et al. Initial report of decreased SARS-CoV-2 viral load after inoculation with the BNT162b2 vaccine. Nat Med 2021;27: 790-2. 